\title{
重氮化合物参与的不对称催化多组分反应
}

\author{
唐 敏 ${ }^{a, b}$ 邢 栋 ${ }^{a, b}$ 蔡茂强 ${ }^{a, b}$ 胡文浩*,, , $b$ \\ ( ${ }^{a}$ 华东师范大学上海分子治疗与新药创制工程技术研究中心 上海 200062)

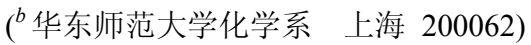

\begin{abstract}
摘要 综述了近年来我们研究小组在重氮化合物参与的不对称催化多组分反应方面的研究进展. 基于对重氮化合物经 由金属卡宾而生成的羟基叶立德、氨基叶立德或活泼离子对中间体的亲电捕捉，以及不同模式的协同催化策略，发展 了不同类型的不对称催化多组分反应，实现了多种高效构建多官能团手性化合物的合成策略.

关键词 重氮化合物; 金属卡宾; 不对称催化; 多组分反应; 协同催化
\end{abstract}

\section{Diazo Compounds-Involved Catalytic Asymmetric Multicomponent Reactions}

\author{
Tang, Min ${ }^{a, b}$ Xing, Dong ${ }^{a, b} \quad$ Cai, Maoqiang ${ }^{a, b} \quad \mathrm{Hu}$, Wenhao $^{*, a, b}$ \\ ( ${ }^{a}$ Shanghai Engineering Research Center of Molecular Therapeutics and New Drug Development, \\ East China Normal University, Shanghai 200062) \\ ( ${ }^{b}$ Department of Chemistry, East China Normal University, Shanghai 200062)
}

\begin{abstract}
This accounts covers the recent advances in diazo compounds-involved catalytic asymmetric multicomponent reactions developed by our research group. Based on the electrophilic trapping of active oxonium ylides, ammonium ylides or zwitterionic intermediates that generated from diazo compounds via metal carbenes, an array of catalytic asymmetric multicomponent reactions have been developed. Cooperative catalytic strategies have been applied to control enantioselectivity of the reactions. These transformations offer efficient and powerful strategies for the synthesis of polyfunctional chiral molecules. Keywords diazo compound; metal carbene; catalytic asymmetric; multicomponent reaction; cooperative catalysis
\end{abstract}

多组分反应因其兼具高原子经济性、高汇聚性、操 作简单、快速高效、易于实现多样性导向合成等特点，一 直以来受到有机化学家的持续关注, 被广泛的应用于从 简单原料出发合成大量结构多样化的复杂分子 ${ }^{[1]}$. 然而, 虽然最早的多组分反应可以追溯至 1850 年即被报道的 Strecker 反应, 并且经典的多组分反应如 Ugi 反应等已 经在二十世纪后期的药物开发研究中大放光彩, 但是不 对称催化的多组分反应却是很少, 这显然无法满足现代 药物化学和化学生物学对多样性结构的手性化合物日 益增长的需求. 究其原因, 在于多组分反应中随着反应 组分的增加, 其反应混乱度呈几何增加, 导致反应的选 择性，尤其是对映选择性控制难以实现.

作为金属卡宾最常见的前体，重氮化合物已经被广
泛的应用在各种过渡金属催化的有机转化之中 ${ }^{[2]}$. 近几 十年来，通过采用不同类型的手性金属配合物作为催化 剂, 重氮化合物参与的不对称催化反应如 $\mathrm{C}-\mathrm{H}$ 插入、 $\mathrm{X}-\mathrm{H}$ 插入 $(\mathrm{X}=\mathrm{O}, \mathrm{N}, \mathrm{Si}$ 等)、环丙烷化、重排反应等，都 得到了广泛的发展, 成为有机化学中有力的合成手段. 近年来, 重氮化合物还被应用到了过渡金属催化的多组 分反应中. 这类反应的发展得益于金属卡宾独特的反应 性质. 首先，电中性的金属卡宾碳原子核外由于拥有空 轨道, 具有一定的亲电性, 能够接受含有孤电子对的富 电子基团的类似亲核进攻而生成叶立德或离子对中间 体; 同时，由于吸纳了多余电子，使原本亲电的金属卡 宾极性发生反转, 转变为新的亲核物种, 能够进一步与 亲电试剂反应，从而实现了一类高效的三组分反应

\footnotetext{
*E-mail: whu@chem.ecnu.edu.cn

Received April 3, 2014; revised May 21, 2014; published online June 9, 2014

Project supported by the National Natural Science Foundation of China (Nos. 21125209, 21332003), the Ministry of Science and Technology of China (No. 2011CB808600) and the Science and Technology Commission of Shanghai Municipality (No. 12JC1403800).

国家自然科学基金(Nos. 21125209, 21332003)、科技部杰出青年基金(No. 2011CB808600)和上海市科委重点基础研究基金(No. 12JC1403800)资助项目.
} 
(Scheme 1) $)^{[3]}$ 金属卡宾这种先亲电再亲核的极性反转 特质，和经典的 Ugi 反应中异腈类化合物的作用(亲电 和亲核反应均发生在同一碳原子上)有相似之处, 因此 在多组分反应中具有得天独厚的优势. 另外, 在这种反 应模式下，通过采用手性的金属催化剂、或者利用手性 催化剂对亲电试剂的活化, 可以实现不对称催化的多组 分反应，这是异腈类化合物所参与的多组分反应难以实 现的. 在本专题中, 将对本课题组在该领域开展的研究 工作, 以及其他研究小组在该领域的一些进展进行小 结.

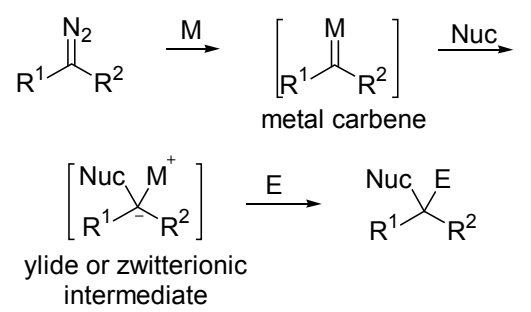

Scheme 1

\section{1 重氮化合物参与的多组分反应的初步探索}

重氮化合物最早应用于多组分反应的例子，应该是 经由金属卡宾实现的 1,3-偶极环加成反应. 在此类反应 中, 重氮化合物首先被金属催化剂分解生成金属卡宾, 金属卡宾随后与羰基或亚胺化合物上的氧/氮孤对电子 反应生成湠基叶立德或亚甲胺叶立德中间体，该类中间 体是很好的 1,3-偶极体, 可以和不同类型的亲偶极体发 生环加成反应, 得到五元氧/氮杂环化合物(Scheme 2) ${ }^{[4]}$. 该类基于 1,3-偶极环加成的多组分反应早在 20 世纪初 即被化学家所发现, 并随后被广泛地用于合成各种氧/ 氮杂环化合物, 然而一个世纪以来, 此类反应在不对称 催化方面却一直未取得很大的进展 ${ }^{[5]}$. 这也是重氮化学 中至今仍值得持续探索的领域之一.

另一方面，对于重氮参与的经由活泼中间体捕捉的 多组分反应逐渐得到了化学家的认识. 该类反应最早的

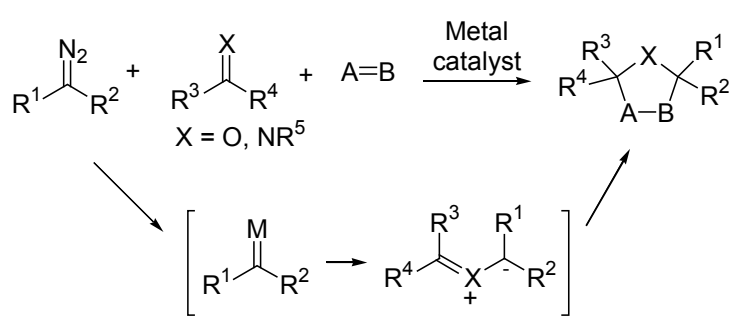

Scheme 2

例子来自于 2001 年我们研究小组 ${ }^{[6]}$ 在开展的重氮化合 物对亚胺的氮杂环丙烷化工作中所发现的一个副反应 (Scheme 3). 当采用对硝基苯甲醛和对硝基苯胺作为亚 胺的前体时, 除了经由亚甲胺叶立德中间体(path A)得 到了意料之中的氮杂环丙烷产物, 和经由氨基叶立德中 间体(path B)生成的 $\mathrm{N}-\mathrm{H}$ 插入副产物之外，我们还意外 地发现了一个来自于对硝基苯胺、重氮化合物和亚胺的 三组分产物(path C).

对于这一三组分产物的生成, 我们推测的机理如 下: 首先, 金属催化剂分解重氮化合物生成金属卡宾中 间体和一分子氮气; 紧接着, 具有空轨道的缺电卡宾碳 从对硝基苯胺带一对孤电子的氮原子处获得电子, 使得 原本电中性的卡宾碳发生极性反转, 从先前的亲电性转 变为此时的亲核性，而氮原子由于电子的给出，便带上 部分正电荷, 这样就生成了一个 1,2-偶极体, 即氨基叶 立德中间体，该中间体一方面可以发生质子的 1,2-迁移 得到 $\mathrm{N}-\mathrm{H}$ 插入产物(path B), 另一方面在具有一定程度 亲电性的物质存在下(比如: 亚胺中带正电性的亚胺碳 原子), 在苯胺氮原子上的质子发生 1,2-迁移到叶立德碳 上之前, 叶立德碳就先被亲电体所捕捉, 最终发生 “竞 争后相对被动的质子迁移延迟” 历程得到三组分产物 (path C). 整个过程中, 由于质子 1,2-迁移和亲电捕捉之 间的竞争, 我们认为, 三组分反应是否能成功发生以及 进行的程度如何, 关键取决于亲电试剂是否有足够的活 性来捕捉叶立德中间体以有效抑制 1,2-质子迁移历程.

与氨基叶立德的形成与捕捉类似，醇或水分子上的

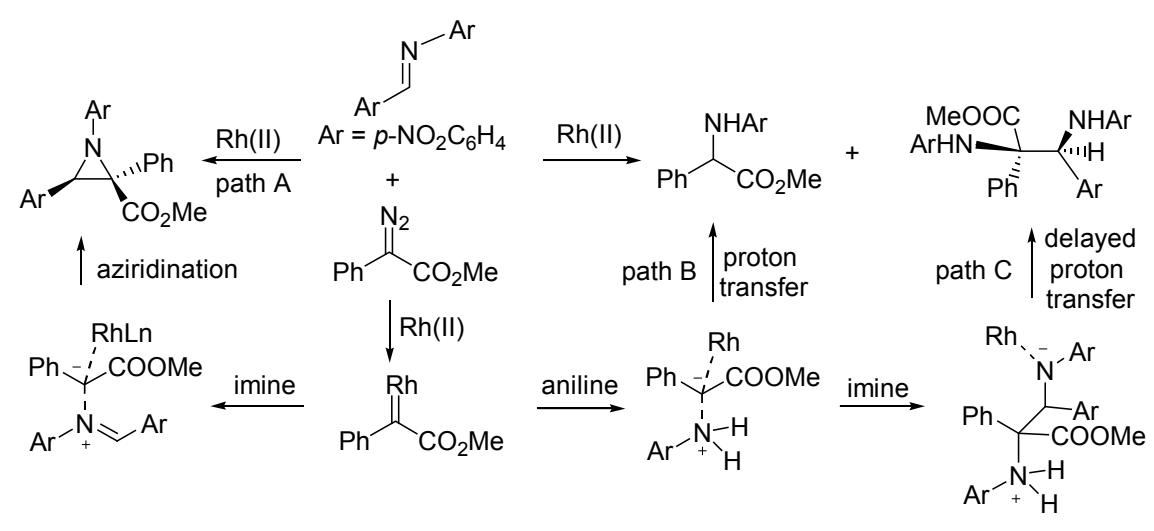

Scheme 3 
氧原子也带有一对孤对电子, 同样可以与金属卡宾作 用，生成着基叶立德中间体，其性质与氨基叶立德中间 体类似，可以发生 1,2-质子迁移得到 $\mathrm{O}-\mathrm{H}$ 插入产物, 而在亲电试剂的存在下, 也有可能通过竞争性的亲电捕 捉历程来延迟 1,2-质子迁移历程, 并实现三组分反应. 当我们采用亚胺或芳香醛等亲电捕体时, 也成功实现了 对羟基叶立德中间体的高效捕捉 ${ }^{[8 a]}$. 这一基于叶立德中 间体捕捉的策略为我们进一步开展的重氮参与的多组 分反应研究开启了一扇大门. 随后, 我们研究小组陆续 实现了消旋条件下其他亲电试剂对活泼氨基和羟基叶 立德中间体的捕捉 (Scheme 4) ${ }^{[7 \sim 9]}$. 为合成不同类型的 双胺类、氨基醇、邻二醇类化合物提供了一种有效的策 略.

Scheme 4 描述了基于氨基或羟基叶立德中间体捕 捉的三组分反应的一般历程. 随着消旋反应研究的深 入, 我们也认识到, 叶立德中间体虽然活泼, 但如果我 们从其前体(金属卡宾)和捕体(亲电试剂)入手来实现不 对称催化控制是有可能的. 前者的关键在于是否可以通 过手性金属催化剂来生成手性的叶立德中间体; 而后者 的关键在于是否可以通过合适的手性催化剂来控制亲 电试剂, 以实现捕捉过程中的不对称催化控制. 本文随 后就对这两方面实现的不对称催化反应进行总结.

\section{2 基于活泼羟基叶立德中间体捕捉的不对称催 化多组分反应}

基于消旋条件下实现的上述研究，我们开始尝试实 现不对称催化的该类多组分反应. 然而, 尽管不同结构 的手性金属配合物已经广泛用于催化重氮化合物参与 的各种反应，但我们采用手性金属催化剂来实现不对称 催化控制的尝试却一直未能取得进展. 为此, 我们将目
光转向了新的策略. 在不对称催化领域, 针对不同类型 的亲电试剂如亚胺; 活泼醛酮等底物的不对称控制而实 现的各种有机转化十分普遍, 不同类型的 Lewis 酸催化 剂、有机小分子催化剂等都在该领域得到了广泛的发展. 为此, 我们考虑引入手性的共催化剂，期望通过对第三 组分亲电试剂的相互作用而实现此类多组分反应的不 对称催化控制.

\section{1 金属/手性磷酸共催化的 Mannich 类型不对称多组 分反应}

2008 年, 我们研究小组和龚流柱小组 ${ }^{[10]}$ 合作报道 了第一例重氮参与的基于捕捉活泼中间体的不对称催 化多组分反应. 该反应以芳基重氮酯为卡宾前体，在金 属铑催化下与 9-葸醇生成活泼着基叶立德中间体，随后 被芳基亚胺捕捉，得到目标三组分产物. 该反应采用手 性磷酸做为共催化剂, 实验结果表明, 手性磷酸能够很 好的和铑金属催化剂兼容，通过和芳基亚胺之间的氢键 作用，很好地调控了该反应的非对映及对映选择性，能 够以高达 98\%的产率、99：1 的非对映选择性和 99\%的 对映选择性得到目标三组分产物(Eq. 1).

对于手性磷酸的作用，一般认为磷酸的质子端首先 与亚胺作用形成氢键, 从而活化亚胺, 另一方面, 磷酸 的磷羰基与羟基叶立德中间体羟鎓氧上的氢发生氢键 相互作用，在两个化学键的共同作用下，叶立德中间体 与亚胺之间的距离被拉近，延迟了质子的转移速度，并 促进了捕捉历程的发生. 同时我们认为，质子所经历的 不仅仅是转移速率的延迟, 更可能经历了从非手性源到 手性源的转移或交换, 最终落于亚胺氮上, 完成整个催 化循环(Scheme 5).

在此共催化条件下，采用芳香醛和苯胺为原料，原 位生成亚胺之后和重氮及醇反应，可较好地实现了一类

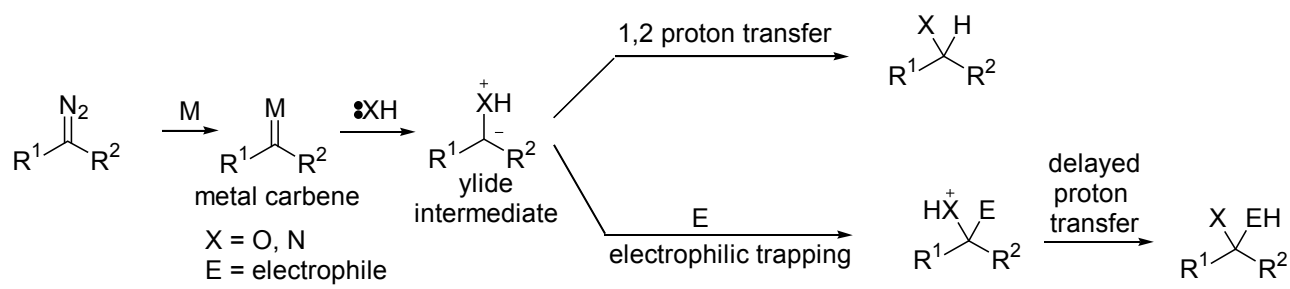

Scheme 4

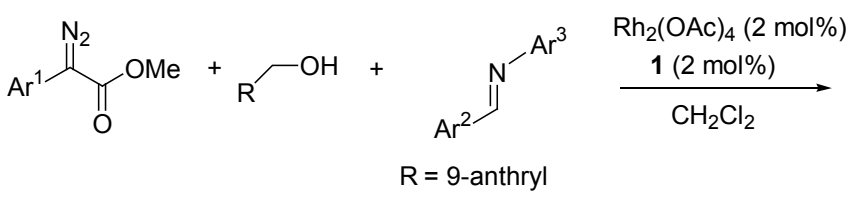




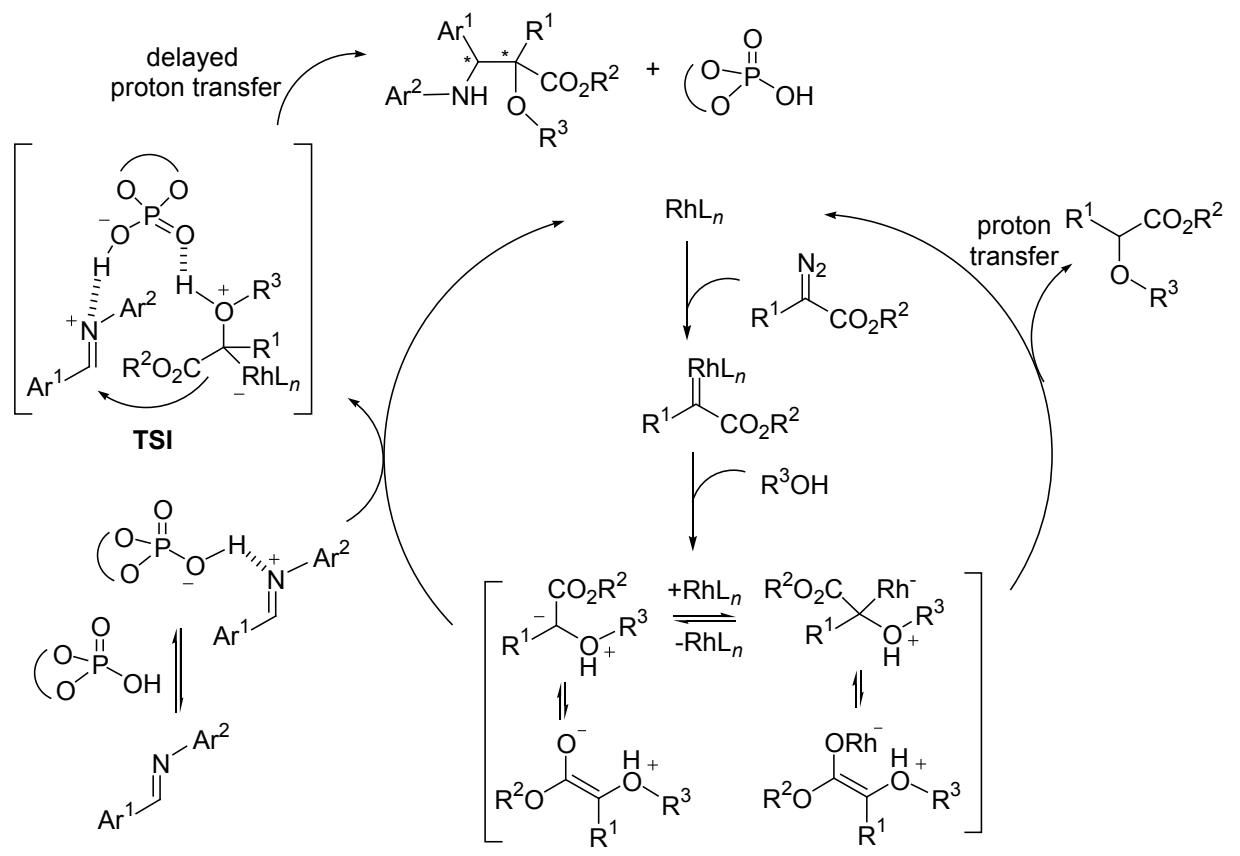

Scheme 5

不对称催化的四组分反应(Eq. 2) ${ }^{[11]}$.

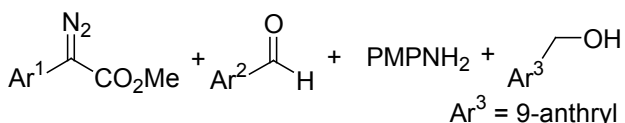

$$
\begin{aligned}
& \mathrm{Rh}_{2}(\mathrm{OAc})_{4}(2 \mathrm{~mol} \%) \\
& 1(2 \mathrm{~mol} \%) \\
& \text { DCM, }-20{ }^{\circ} \mathrm{C}
\end{aligned}
$$

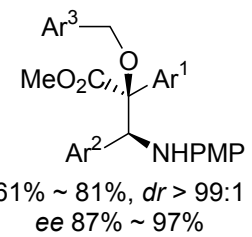

除了醇类化合物, 还可采用水为羟基叶立德中间体 的氧源, 在醋酸铑和手性磷酸的共催化作用下, 和芳基 重氮酸酯与芳香亚胺反应. 通过笁选手性磷酸催化剂, 发现采用 3,3'-(三苯基硅基)取代的手性磷酸 2 时, 可以 以高达 $98: 2$ 的非对映选择性和 $97 \%$ 的对映选择性得到 目标三组分产物(Eq. 3$)^{[12]}$. 同时, 我们还首次将重氮源 拓展到了烷基取代的重氮, 但遗憾的是, 只能以中等的 立体选择性得到目标三组分产物(Eq. 4).

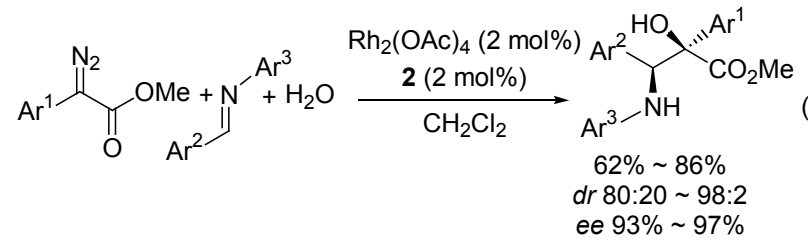

经过进一步的探索, 我们还发展了一例由 $\alpha$-酮羰基 重氮、水、苯胺和乙醛酸酯出发的不对称催化四组分反 应 $^{[13]}$. 手性磷酸 2 可以对苯胺和乙醛酸酯原位生成的亚 胺进行有效的活化, 从而以理想的收率和立体选择性实 现目标 $\beta$-差基- $\alpha$-氨基酸酯的合成(Eq. 5).
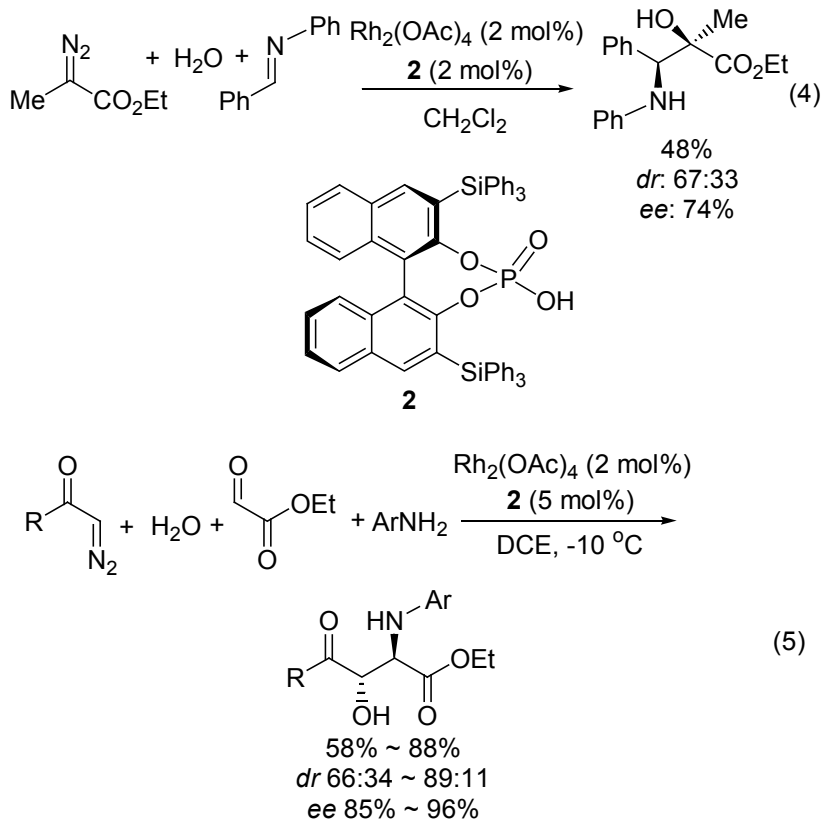

为了进一步扩展该类反应的普适性并体现其合成 价值，接下来我们 ${ }^{[14,15]}$ 进一步研究了不同的重氮化合物 对该类反应的适应性. 当用重氮乙酸酯(EDA)或类似的 苯乙酮重氮作为卡宾源时，通过篎选不同的手性磷酸催 化剂, 分别实现了芳香亚胺对对应的活泼差基叶立德中 间体的捕捉，得到了相应的三组分产物，并且都取得了 理想的收率和中等至好的立体选择性(Scheme 6). 同时, 从重氮乙酸酯和芐醇对应的三组分产物出发, 经过简单 的衍生成功合成了紫杉醇侧链, 进一步证明了该反应的 高效和合成价值(Scheme 6). 


$$
\prod_{\mathrm{O}}^{\mathrm{N}_{2}} \mathrm{OR}^{1}+\mathrm{Ar}^{2} \mathrm{Jl}^{-\mathrm{Ar}^{3}}+\mathrm{Ar}^{1} \mathrm{CH}_{2} \mathrm{OH} \frac{\mathrm{Rh}_{2}(\mathrm{OAc})_{4}(2 \mathrm{~mol} \%)}{\mathbf{3}(5 \mathrm{~mol} \%)}
$$$$
\begin{array}{cc}
\mathrm{X}=4-\mathrm{FC}_{6} \mathrm{H}_{4} \\
\prod_{\mathrm{O}}^{\mathrm{N}_{2}} \mathrm{Ar}^{1}+\mathrm{Ar}^{3}{ }^{\mathrm{N}-\mathrm{Ar}^{4}}+\mathrm{Ar}^{2} \mathrm{CH}_{2} \mathrm{OH} & \frac{\mathrm{Rh}_{2}(\mathrm{OAc})_{4}(2 \mathrm{~mol} \%)}{\mathbf{4}(5 \mathrm{~mol} \%)} \\
\text { toluene }
\end{array}
$$<smiles>C#CC(=O)OC(C(=O)O)[C@@H](NC(=O)c1ccccc1)c1ccccc1</smiles>

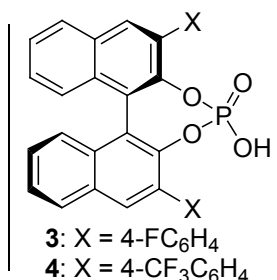

\section{Scheme 6}

\section{2 金属/Lewis 酸共催化的 aldol 类型不对称催化多组} 分反应

除了采用亚胺为亲电试剂对活泼着基叶立德中间 体实现的 Mannich 类型的捕捉, 我们研究小组 ${ }^{[16]}$ 还实现 了芳香醛出发的 aldol 类型的捕捉. 并且, 通过采用 BINOL-衍生的手性锆配合物为共催化剂, 实现了第一 例金属/Lewis 酸共催化的不对称 aldol 类型三组分反应. 在该反应中, 芳基重氮酯在醋酸铑作用下生成铑卡宾, 并与苠醇反应生成差基叶立德中间体; 另一方面, 手性 锆配合物与叶立德捕捉体(芳香醛)生成有效的络合物. 在手性锆的络合作用下, 芳香醛对着基叶立德中间体的 捕捉过程可以实现很好的手性控制. 该反应底物范围较 广, 产物的产率、立体选择性均较好(Scheme 7).

\section{3 金属/Lewis 酸共催化的 Michael 类型不对称催化}

\section{多组分反应}

随后, 利用金属/Lewis 酸共催化策略, 我们研究小
组 ${ }^{[17]}$ 又进一步实现了首例不对称催化的 Michael 类型多 组分反应. 该反应以苯基重氮酸酯为卡宾前体，水为羟 基叶立德源, 以 $\alpha, \beta$-不饱和酮类化合物为亲电试剂. 采 用手性双恶唑啉配体(BOX)配位的二价锌可以和 $\alpha, \beta$ 不饱和酮类化合物络合，从而实现有效的不对称控制， 最终以 $61 \% \sim 68 \%$ 的产率, $86: 14 \sim 99 ： 1$ 的非对映选 择性以及 $85 \%$ \% $99 \%$ 的对映选择性得到目标三组分产物 (Scheme 8).

\section{3 基于活泼氨基叶立德中间体捕捉的不对称催 化多组分反应}

尽管早在 2001 年我们便实现了消旋条件下基于氨 基叶立德中间体捕捉的多组分反应，但直到 2011 年，我 们 $^{[18]}$ 才实现了该类反应的不对称催化控制，采取同样 的金属/手性磷酸共催化策略，从芳基重氮酸酯、烷氧酰 胺和芳香亚胺出发, 得到了对应的 $\beta$-氨基- $\alpha$-氨基酸酯

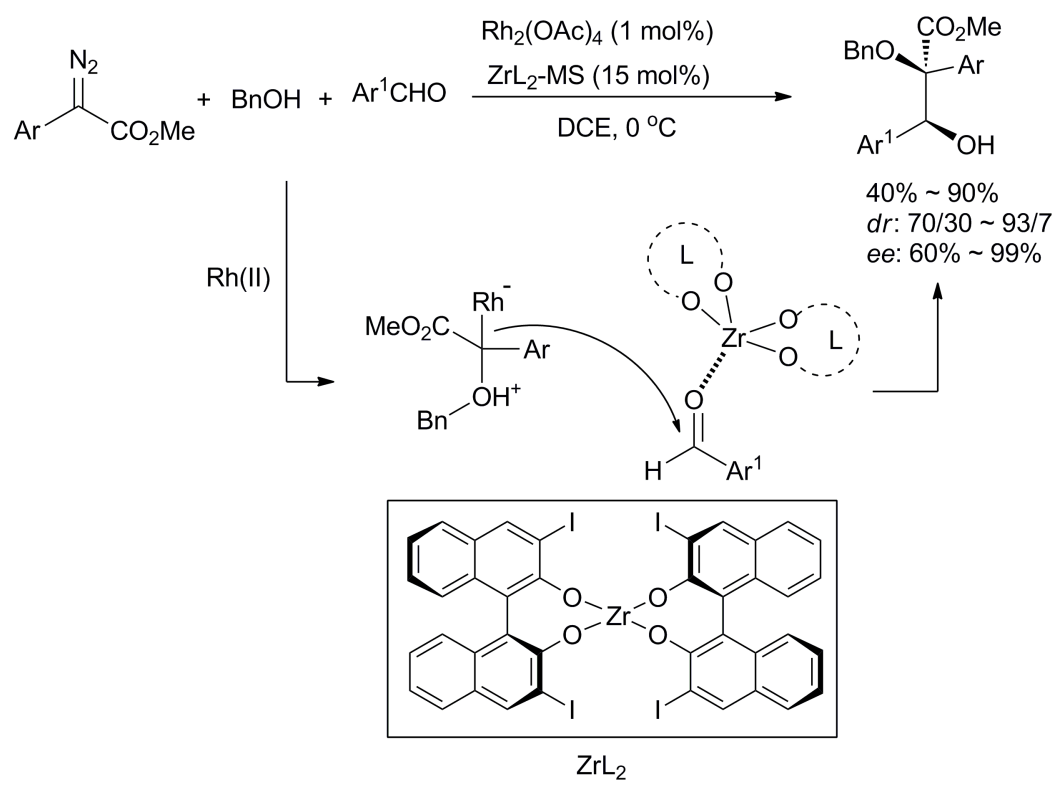

Scheme 7 


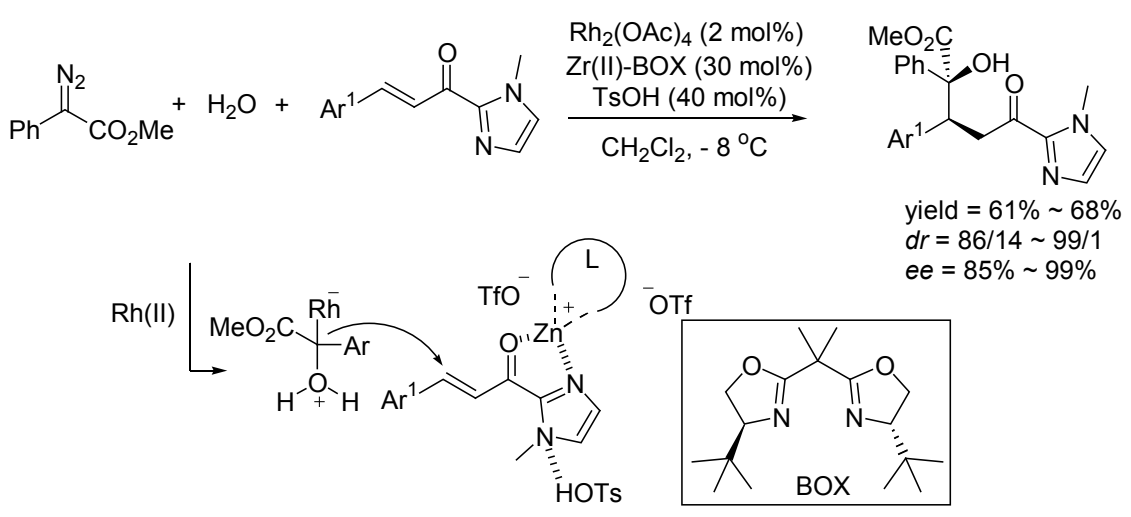

Scheme 8

类三组分产物. 与羟基叶立德中间体的捕捉稍有不同， 该反应需要加入酒石酸作为添加剂, 可以有效促进反应 的选择性. 同时, 通过手性磷酸催化剂的选择, 可实现 该反应的非对映选择性的调控(Eq. 6). 我们认为, 反应 的不对称控制仍是通过亚胺对着基叶立德捕捉相似的 双氢键的活化作用而实现的(TSII). 之后，在类似的共 催化条件下, 我们进一步 ${ }^{[19]}$ 将底物类型拓展至了芳香 基磷酰胺和醛酸酯芳亚胺, 从而发展了一类不对称合成 2,3-二氨基丁二酸衍生物的方法.

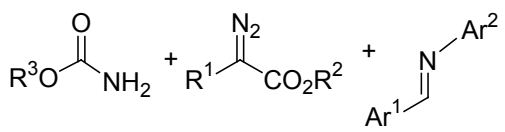

$$
\begin{aligned}
& \mathrm{Rh}_{2}(\mathrm{OAc})_{4}(2 \mathrm{~mol} \%) \\
& \text { chiral PPA (5 mol\%) } \\
& L \text {-tartaric acid }(20 \mathrm{~mol} \%) \\
& \text { toluene, }-20{ }^{\circ} \mathrm{C} \\
& \mathrm{R}^{3} \mathrm{O} \\
& \text { (- }
\end{aligned}
$$

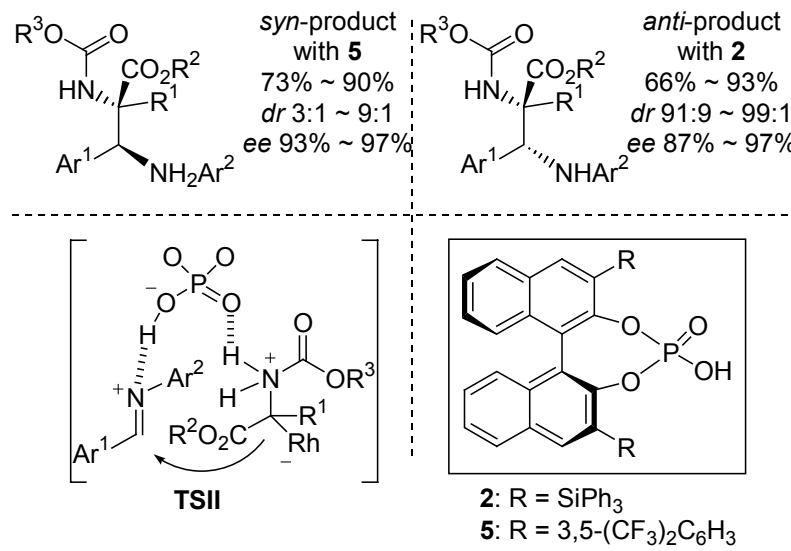

之后, 我们研究小组 ${ }^{[20]}$ 进一步报道了一例从芳香 胺、芳基重氮酯和醛酸酯出发的三分子四组分反应. 同 样采用醋酸铑/手性磷酸共催化体系, 一分子的芳香胺 首先与自重氮生成的铑卡宾反应生成氨基叶立德中间 体, 另一方面, 另一分子的芳香胺和醛酸酯反应生成亚 胺, 亚胺在手性磷酸的活化作用下对氨基叶立德中间体
发生亲电捕捉, 得到四组分产物. 通过系统的条件篮选, 该反应可以以理想的收率，中等至好的非对映选择性和 对映选择性生成一系列 $\alpha, \beta$-双氨基酸类衍生物(Eq. 7).

$$
\begin{aligned}
& \underset{2.2 \text { equiv. }}{\mathrm{ArNH}_{2}}+\mathrm{H}_{\mathrm{CO}_{2} \mathrm{Et}}^{\stackrel{\mathrm{O}}{\mathrm{O}}}+\underset{\mathrm{R}}{\stackrel{\mathrm{O}}{\mathrm{H}}} \mathrm{N}_{2} \\
& \frac{\mathrm{Rh}_{2}(\mathrm{OAc})_{4}(2 \mathrm{~mol} \%)}{1(5 \mathrm{~mol} \%)} \\
& \mathrm{ArHN}{ }_{\mathrm{H}}^{\mathrm{NHAr}} \mathrm{CO}_{2} \mathrm{Et} \\
& 50 \% \sim 86 \% \\
& \text { dr 67:33 95:5 } \\
& \text { ee } 67 \% \sim 97 \%
\end{aligned}
$$

2013 年，中国科技大学龚流柱研究小组 ${ }^{[21]}$ 报道了 一例由苯胺、3-重氮氧化吲哚和醛酸酯出发的不对称催 化三组分反应. 在醋酸铑和手性磷酸的共催化作用下, 首次实现了对氨基叶立德中间体 aldol 类型的不对称捕
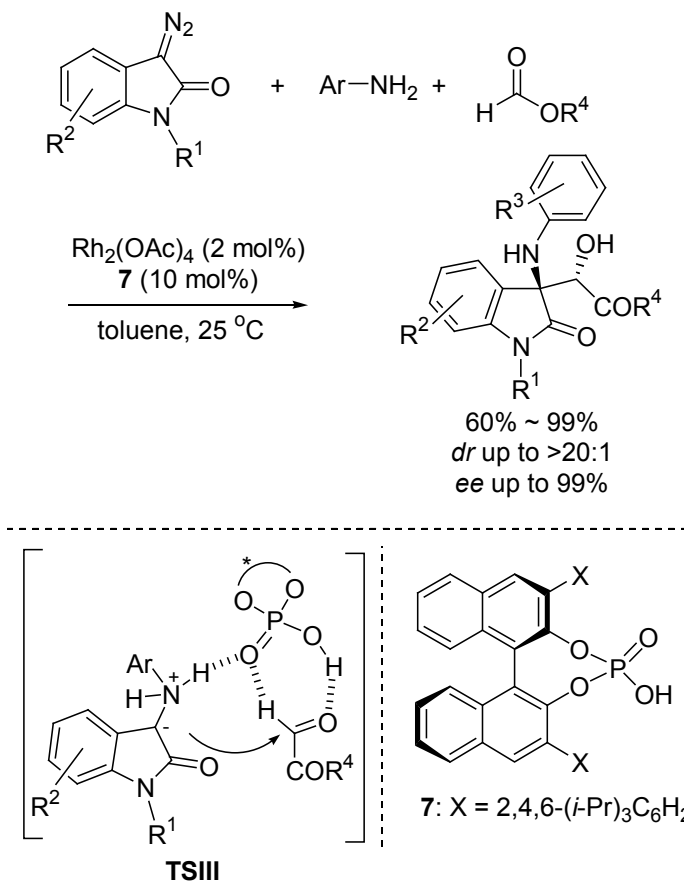

7: $\mathrm{X}=2,4,6-(i-\operatorname{Pr})_{3} \mathrm{C}_{6} \mathrm{H}_{2}$ 
捉，合成了一系列 3,3-双取代的氧化吲哚类化合物(Eq. 8). 该反应的不对称控制的关键在于手性磷酸与醛酸酯 以及氨基叶立德中间体之间生成的双重氢键的相互作 用(TSIII), 此反应生成的 3,3'-双取代氧化吲哚类化合物 广泛存在于生物活性分子中, 为拓展此类不对称催化的 多组分反应在药物化学中的应用有一定的促进作用.

2012 年, 香港大学的支志明研究小组 ${ }^{[22]}$ 报道了一 例手性铑催化剂催化的芳香胺、芳基重氮磷酸酯和芳香 醛的不对称催化三组分反应. 在该反应中, 芳基重氮磷 酸酯首先在手性铑的催化下生成手性铑卡宾, 进而与芳 胺反应生成氨基叶立德中间体, 体系中的芳香醛对该中 间体进行亲电捕捉, 生成了手性的目标三组分产物 (Scheme 9). 这一报道与之前的例子不同之处在于采用 单一的手性金属催化剂便实现了理想的不对称催化控 制, 说明了在活泼叶立德中间体生成过程中是有可能将 金属卡宾的手性得以保持并传递至进一步的转化中. 因 此, 为该研究领域提供了一种新的策略, 可以充分利用 业已十分丰富的手性铑催化剂资源, 发展新型的不对称 催化多组分反应.
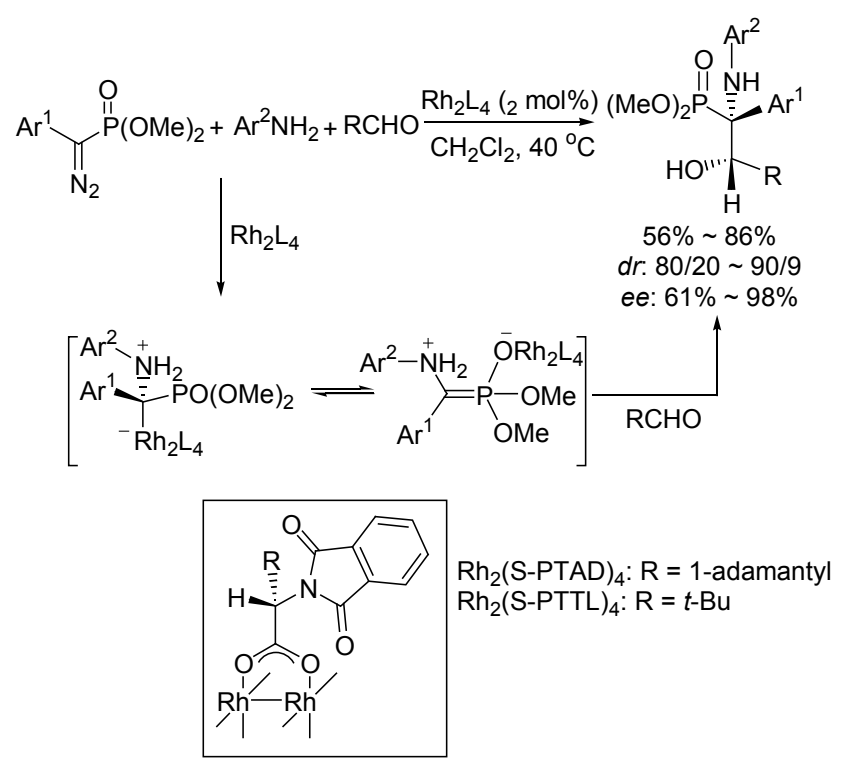

Scheme 9

\section{4 基于活泼离子对中间体捕捉的不对称催化多}

\section{组分反应}

含有烯胺单元的分子如吲哚、吡啶及其他类型的含 氮杂环分子可以和金属卡宾反应，在烯胺单元上的氮原 子的孤对电子的稳定作用下，生成一类离子对中间体. 该类中间体可以发生质子转移, 从而实现金属卡宾对该 类底物的 $\mathrm{C}-\mathrm{H}$ 官能团化, 这是有机合成中发展十分成 熟的一类反应. 基于对活泼叶立德中间体进行亲电捕捉 的研究, 我们研究小组 ${ }^{[23]}$ 于 2012 年实现了基于对此类
活泼离子对中间体进行捕捉的三组分反应. 在醋酸铑和 手性磷酸的共催化条件下，吲哚、芳基重氮酯和芳基亚 胺发生高效的不对称催化反应，生成了一系列 3-取代的 吲哚类化合物. 该反应仍然以铑卡宾的形成为开端，随 后吲哚与金属卡宾反应生成活泼离子对中间体，同时， 被手性磷酸活化的亚胺对该中间体进行亲电捕捉, 经由 过渡态 TSIV, 成功实现立体选择性控制, 得到目标三 组分产物(Scheme 10). 该例反应不同于叶立德中间体 捕捉时率先生成 $\mathrm{C}-\mathrm{X}(\mathrm{X}=\mathrm{O}, \mathrm{N})$ 键, 而实现了两根 $\mathrm{C}-\mathrm{C}$ 键的生成, 进一步丰富了此类不对称催化反应的 应用范围. 基于醋酸铑和手性磷酸的协同催化策略, 我 们还发展了一例高效地利用重氮化合物对吲哚进行碳 氢插入的反应 ${ }^{[24]}$.
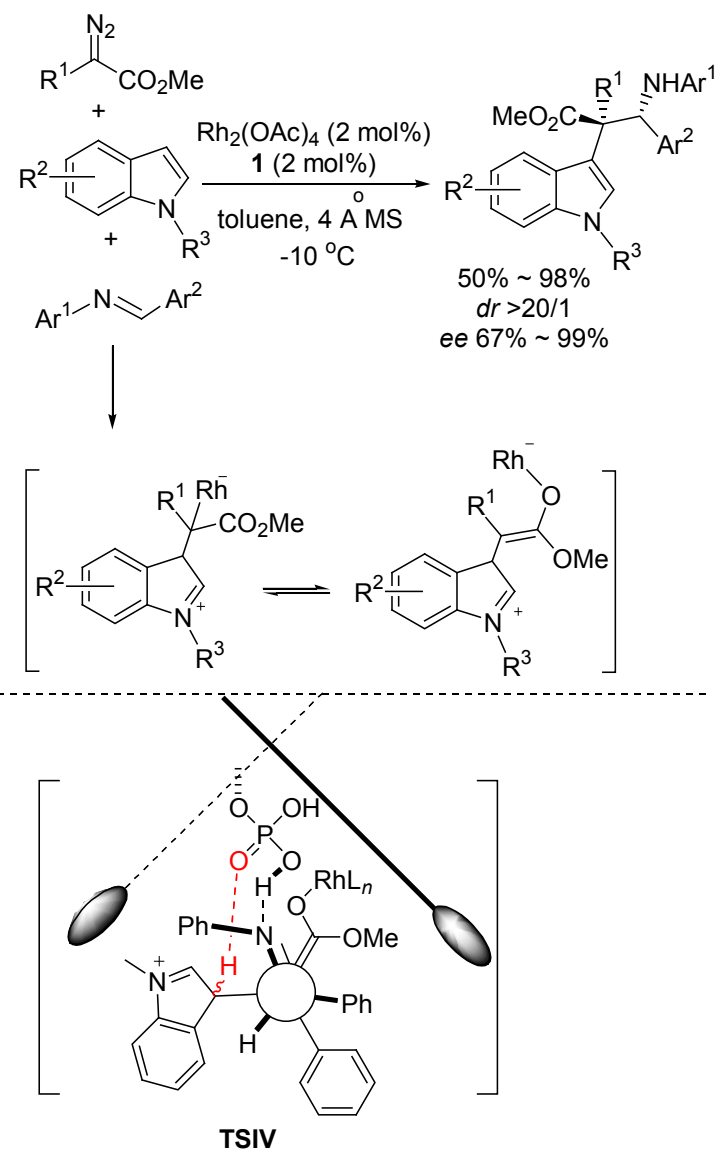

Scheme 10

从以上描述的反应可以看到, 吲哚可以很好地作 为活泼离子对中间体的来源, 发生新型的不对称催化多 组分反应，那其他类型的含氮杂环分子是否也可以发生 类似的反应呢? 金属卡宾经由离子对中间体对吡咯选 择性的进行 2 位或 3 位的 $\mathrm{C}-\mathrm{H}$ 官能团化的反应早有报 道 ${ }^{[25]}$. 为此, 我们认为通过合适的催化体系和亲电捕捉 试剂的选择, 可以实现吡咯参与的不对称催化三组分反 应. 然而, 在醋酸铑/手性磷酸的共催化作用下，由吡 
咯、芳基重氮酯和芳基亚胺出发的三组分反应未能取得 理想的结果. 于是, 我们将目光转向了其他类型的金属 催化剂, 经过系列篎选, 我们发现烯丙基钯/手性磷酸的 催化组合可以有效地实现上述反应的立体控制, 得到一 系列 2-取代的吡咯衍生物. 另外当采用不同取代的手性 磷酸催化剂以及加入酒石酸为添加剂时, 该反应还可以 实现非对映选择性的完全反转(Scheme 11) ${ }^{[26]}$. 我们认 为该反应的机理包括钯卡宾中间体的生成、吡咯和卡宾 反应得到离子对中间体, 另一方面亚胺在手性磷酸的活 化作用下对离子对中间体进行亲电捕捉, 实现不对称控 制并得到目标三组分产物. 有趣的是, 在机理研究过程 中, 我们发现预先制备的手性磷酸钯配合物本身同样可 以催化该三组分反应, 并取得很好的立体控制, 初步说 明手性钯卡宾的手性在随后的转化中得到了保持和传 递. 进一步验证了支志明研究小组所报道的采用单一金 属催化剂对重氮参与的多组分反应进行不对称催化控 制的可行性.

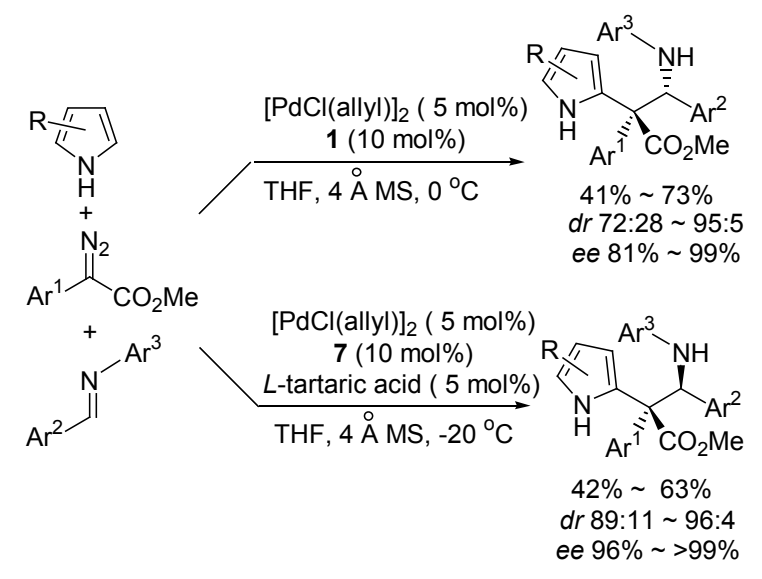

\section{Scheme 11}

\section{5 总结与展望}

经过十几年的发展, 重氮化合物参与的经由活泼 中间体捕捉的多组分反应已经得到了较系统的发展. 这 类新的反应策略在概念上对重氮化学是很好的完善. 通 过采取过渡金属/手性磷酸共催化体系、单一手性过渡金 属催化剂、过渡金属/手性 Lewis 酸共催化体系等, 我们 和其他研究小组分别实现了对活泼羟基叶立德、氨基叶 立德和离子对中间体的亲电捕捉, 发展了不同类型的不 对称催化多组分反应，填补了经典多组分反应在实现不 对称催化控制方面的空白. 另外从合成的角度看, 也提 供了一些十分高效和实用的合成手性多取代复杂化合 物的方法.

可以看到，该类反应在底物的适用性和在合成中的 应用性方面还存在一定的不足, 因此进一步的研究应集
中在拓宽该策略的应用范围，进一步体现其合成价值. 同时，关于此类多组分反应的机理方面的研究工作开展 较少, 这也将是我们今后努力的方向之一, 我们相信随 着对其研究的深入, 必将有更多更有价值的不对称催化 多组分反应在将来被发现.

\section{作者简介}

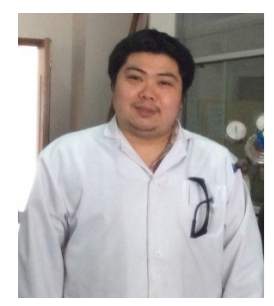

唐敏, 2004 年 7 月毕业于西南师范大学化学系; 2004 年 8 月至 2006 年 7 月就职于中国科学院成都有机 化学研究所, 实习助理研究员; 2006 年 8 月至 2008 年 7 月就职于上海清松制药有限公司，从事工艺优化; 2008 年 9 月至 2010 年 8 月, 就读于华东师范大学生命科学学 院, 基因组学专业; 2010 年 8 月至今, 就读于华东师范 大学科学与技术跨学科高等研究院, 攻读有机化学博士 学位.

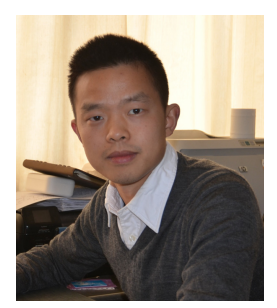

邢栋, 2003 年和 2006 年分别在华东师范大学获本 科和硕士学位. 2011 年于香港大学获博士学位, 2012 年 加入华东师范大学上海分子治疗与工程技术研究中心, 任助理研究员.

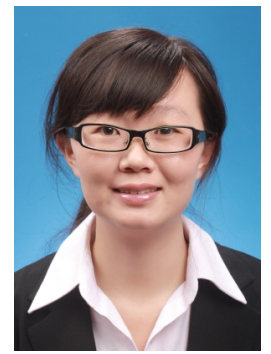

蔡茂强, 2011 年 7 月毕业于四川大学化学学院，同 年 9 月入学华东师范大学科学与技术跨学科高等研究院 攻读硕士学位. 


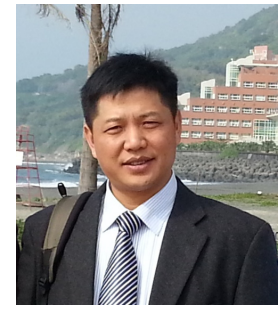

胡文浩, 1987 年四川大学本科毕业. 1998 年于香港 理工大学获博士学位. 1998 2001 年在美国亚利桑那大 学化学系做博士后. 2002 2006 年分别在美国 GeneSoft 和施贵宝制药公司工作. 2006 年回国到华东师范大学任 教, 现任上海分子治疗与新药创制工程技术研究中心主 任, 2011 年获国家杰出青年基金.

\section{References}

[1] (a) Zhu, J.; Bienaymé, H. Multicomponent Reactions, Wiley, Weinheim, Germany, 2005.

(b) Ramón, D. J.; Yus, M. Angew. Chem., Int. Ed. 2005, 44, 1602.

(c) Dömling, A. Chem. Rev. 2006, 106, 17.

(d) Ruijter, E.; Scheffelaar, R.; Orru, R. V. A. Angew. Chem., Int. Ed. 2011, 50, 6234.

[2] (a) Li, A.-H.; Dai, L.-X.; Aggarwal, V. K. Chem. Rev. 1997, 97, 2341 .

(b) Davies, H. M. L.; Manning, J. R. Nature 2008, 451, 417.

(c) Sun, X.-L.; Tang, Y. Acc. Chem. Res. 2008, 41, 937.

(d) Zhang, Z.; Wang, J. Tetrahedron 2008, 64, 6577.

(e) Zhu, S.-F.; Zhou, Q.-L. Acc. Chem. Res. 2012, 45, 1365.

(f) Li, Y.; Huang, C.; Xu, P.; Zhang, Y.; Wang, J. Acta Chim. Sinica 2012, 70, 2024 (in Chinese).

(李玉叶, 黄重行, 许鹏飞, 张艳, 王剑波, 化学学报, 2012, 70, 2024.)

[3] Guo, X.; Hu, W. Acc. Chem. Res. 2013, 46, 2427.

[4] Nair, V.; Rajesh, C.; Vinod, A. U.; Bindu, S.; Sreekanth, A. R.; Mathen, J. S.; Balagopal, L. Acc. Chem. Res. 2003, 36, 899

[5] Staffan, T.; Peter, S. Adv. Synth. Catal. 2006, 348, 2421.

[6] Doyle, M. P.; Hu, W.; Timmons, D. J. Org. Lett. 2001, 3, 933.

[7] Wang, Y.; Zhu, Y.; Chen, Z.; Mi, A.; Hu, W.; Doyle, M. P. Org. Lett. 2003, 5, 3923.
[8] (a) Wang, Y.; Chen, Z.; Mi, A.; Hu, W. Chem. Commun. 2004 2486 .

(b) Guo, X.; Yue, Y.; Hu, G.; Zhou, J.; Zhao, Y.; Yang, L.; Hu, W. Synlett 2009, 2109

(c) Zhu, Y.; Zhai, C.; Yang, L.; Hu, W. Eur. J. Org. Chem. 2011, 1113 .

(d) Zhang, X.; Ji, J.; Zhu, Y.; Jing, C.; Li, M.; Hu, W. Org. Biomol. Chem. 2012, 10, 2133.

[9] (a) Lu, C.; Liu, H.; Chen, Z.; Hu, W.; Mi, A. Org. Lett. 2005, 7, 83. (b) Han, X.; Jiang, L.; Tang, M.; Hu, W. Org. Biomol. Chem. 2011, 9, 3839 .

(c) Guo, X.; Huang, H.; Yang, L.; Hu, W. Org. Lett. 2007, 9, 4721.

(d) Huang, H.; Guo, X.; Hu, W. Angew. Chem., Int. Ed. 2007, 46, 1337.

[10] Hu, W. H.; Xu, X. F.; Zhou, J.; Liu, W. J.; Huang, H. X.; Hu, J.; Yang, L. P.; Gong, L. Z. J. Am. Chem. Soc. 2008, 130, 7782.

[11] Xu, X.; Zhou, J.; Yang, L.; Hu, W. Chem. Commun. 2008, 6564.

[12] Qian, Y.; Jing, C.; Shi, T.; Ji, J.; Tang, M.; Zhou, J.; Zhai, C.; Hu, W. ChemCatChem 2011, 3, 653.

[13] Qian, Y.; Jing, C.; Liu, S.; Hu, W. Chem. Commun. 2013, 49, 2700.

[14] Qian, Y.; Xu, X.; Jiang, L.; Prajapati, D.; Hu, W. J. Org. Chem. 2010, 75, 7483.

[15] Xu, X.; Qian, Y.; Yang, L.; Hu, W. Chem. Commun. 2011, 47, 797.

[16] Zhang, X.; Huang, H.; Guo, X.; Guan, X.; Yang, L.; Hu, W. Angew. Chem., Int. Ed. 2008, 47, 6647.

[17] Guan, X.; Yang, L.; Hu, W. Angew. Chem., Int. Ed. 2010, 49, 2190.

[18] Jiang, J.; Xu, H.; Xi, J.; Ren, B.; Lv, F.; Guo, X.; Jiang, L.; Zhang, Z.; Hu, W. J. Am. Chem. Soc. 2011, 133, 8428.

[19] Jiang, J.; Ma, X.; Liu, S.; Qian, Y.; Lv, F.; Qiu, L.; Wu, X.; Hu, W. Chem. Commun. 2013, 49, 4238.

[20] Jing, C.; Xing, D.; Qian, Y.; Hu, W. Synthesis 2014, 46, 1348.

[21] Ren, L.; Lian, X.; Gong, L. Chem. Eur. J. 2013, 19, 3315.

[22] Zhou, C.; Wang, J.; Wei, J.; Xu, Z.; Guo, Z.; Low, K.; Che, C. Angew. Chem., Int. Ed. 2012, 51,11376.

[23] Qiu, H.; Li, M.; Jiang, L.-Q.; Lv, F.-P.; Zan, L.; Zhai, C.-W.; Doyle, M. P.; Hu, W.-H. Nat. Chem 2012, 4, 733.

[24] Qiu, H.; Zhang, D.; Liu, S.; Qiu, L.; Zhou, J.; Qian, Y.; Zhai, C.; Hu, W. Acta Chim. Sinica 2012, 70, 2484 (in Chinese). (邱晃, 张丹, 刘顺英, 邱林, 周俊, 钱宇, 翟昌伟, 胡文浩, 化 学学报, 2012, 70, 2484.)

[25] Maryanoff, B. E. J. Org. Chem. 1979, 44, 4410.

[26] Zhang, D.; Qiu, H.; Jiang, L.; Lv, F.; Ma, C.; Hu, W. Angew. Chem., Int. Ed. 2013, 52, 13356. 